\section{Verlust der Sehschärfe war Folge eines Bronchialkarzinoms}

\section{Ein 56-jähriger Mann wurde von seinem Augenarzt mit akutem schmerzhaftem Visusverlust des rechten Auges zur Aufnahme in die Augenklinik eingewiesen. Er berichtete über einen unfreiwilligen Gewichtsverlust von $10 \mathrm{~kg}$ in den letzten 4 Wochen. Eine Schwester des Patienten war wegen eines zentralen Bronchialkarzinoms pneumonektomiert worden.}

In der augenärztlichen Untersuchung fand sich eine entrundete, lichtstarre rechte Pupille. Fundoskopisch zeigte sich eine solide Prominenz im Augapfel.

Das MRT blieb ohne Tumornachweis, allerdings wurde der Verdacht auf eine Aderhautmetastase geäußert. Bei der Primärtumorsuche fand sich in der Thorax-CT ein kleiner peripherer Rundherd im rechten Lungenoberlappen, der maligne imponierte $(\bullet \mathbf{A b b}$.

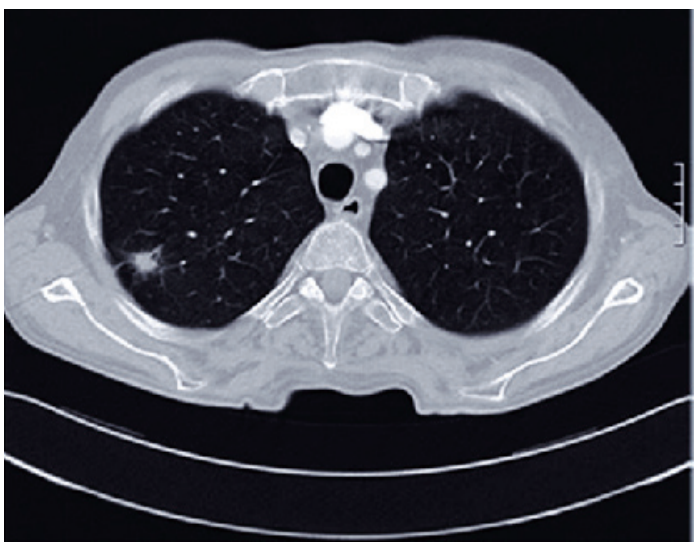

1). Oberbauch-CT, Sonographie des Abdomens sowie Skelettszintigraphie ergaben dagegen keinen Hinweis auf ein Tumorleiden und auch die Bronchoskopie erbrachte keinen wegweisenden Befund.

Abb. $1<$ CT-Thorax mit i.v.-Kontrastmittel. Zwischen Segment 1 und 2 rechts ein unscharf begrenzter, inhomogener Tumor mit Spiculae

\section{Therapie: Enukleation und Resektion des Bronchialkarzinoms}

Die in der interdisziplinären Tumorkonferenz vonseiten der Augenärzte indizierte Enukleation des rechten Auges erfolgte komplikationslos. Der histopathologische Befund beschrieb die R0-Resektion einer intraokularen Bronchialkarzinommetastase. 19 Tage nach der Enukleation wurde mittels rechtsseitiger axilloanteriorer Thorakotomie eine Bisegmentresektion (Segmente 1 und 2) mit systematischer Lymphknotendissektion durchgeführt.

Es lag ein $2 \mathrm{~cm}$ großes, peripheres, überwiegend gering differenziertes Adenokarzinom mit regionärer Lymphangiosis carcinomatosa und 21 tumorfreien Lymphknoten vor

(- Abb. 2). Nach UICC wurde Tumorstadium IV festgelegt (pT1pN0(0/21)pM1R0L1V0G3).
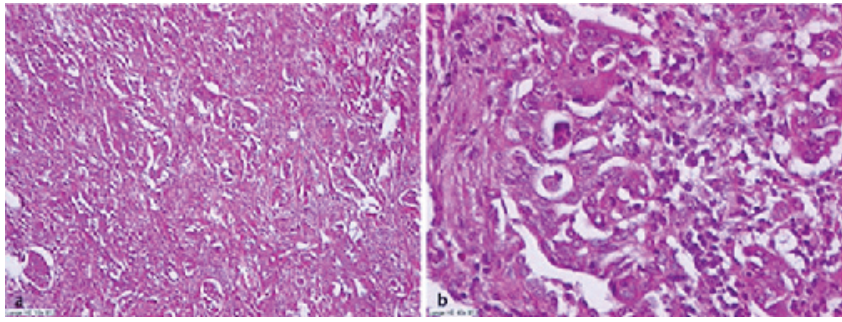

Abb. $2<$ a HE-Lungenpräparat in 10 facher Vergrößerung mit Nachweis eines soliden Tumors.

b Ausschnitt aus Abb. 2a (Vergr. 40:1).

Bei erneuter Vorstellung des Falls in der interdisziplinären Tumorkonferenz wurde aufgrund der Lymphangiosis carcinomatosa im Zentrum des Tumors und der stattgehabten hämatogenen Fernmetastasierung eine adjuvante Chemotherapie empfohlen.

Quelle: Ophthalmologe (2010) 107: 549-552 eine primäre oder sekundäre Fernmetastasierung stark erhöht. Ein multimodales Vorgehen sollte postdiagnostisch und prätherapeutisch interdisziplinär festgelegt werden. Im Falle von solitären mediastinalen Lymphknotenmetastasen, die von der Umgebung gut abgegrenzt sind, kann eine primäre Resektion durchgeführt werden, der eine adjuvante Bestrahlung folgen sollte. In der Regel wird jedoch in dieser Situation sowie bei isoliertem Befall einer Lymphknotengruppe eine präoperative Chemotherapie oder Radiochemotherapie mit anschließender sekundärer Resektion durchgeführt (• Abb. 5). Diese Patienten werden als primär resektabel angesehen und eine sekundäre Resektion ist nach Vorbehandlung parenchymsparend und radikal in der Regel möglich. Die 5-Jahres-Überlebensrate wird mit $10-30 \%$ angegeben. $\mathrm{Pa}$ tienten mit multiplem N2-Befall können gleichermaßen mit einer Kombination aus Strahlentherapie und Chemotherapie (definitive Radiochemotherapie) behandelt werden.

Bei funktionell inoperablen Patienten ist eine definitive Radiochemotherapie indiziert.

Die Nachsorge erfolgt symptomorientiert im 3-Monats-Rhythmus, um palliative Maßnahmen bei Fernmetastasierung (Hirn, Knochen) einleiten zu können, bevor Komplikationen auftreten.

\section{Lungentumor mit fortgeschrittener ip-} silateraler oder kontralateraler Lymphknotenmetastasierung (Stadium IIIA4 und IIIB). Handelt es sich um konfluierende Metastasen über mehrere Lymphknotenregionen, die evtl. vom Primärtumor nicht abgrenzbar sind, gelten die Patienten als primär inoperabel. Das primäre und sekundäre Metastasierungsrisiko ist sehr hoch. Die primäre Therapie besteht hier in einer definitiven simultanen Radiochemotherapie oder (bei Kontraindikation gegen eine Chemotherapie) in der alleinigen definitiven Strahlentherapie. In erfahrenen Zentren kann im Rahmen des Restagings bei Patienten mit günstigem Verlauf (guter Allgemeinzustand und gutes Ansprechen, radikale Resektion ohne Pneumonektomie mög- 